\title{
DESCARTES ET LE THEATRE
}

Jean-Pierre Babin

Ce titre ne doit pas être entendu au sens de "Descartes dramaturge": il ne s'agit pas de ce qu'il y aurait de thêtral dans l'écriture cartésienne, soit dans le style, soit dans les personnages qu'il fait comparaitre, par exemple, mais plutôt de ce que le thêâtre permet à Descartes de penser. De plus, nous ne prétendons pas ici a l'exhaustivité dans le relevé et la prise en compte des réflexions cartésiennes sur le thêahtre: nous nous intéresserons essentiellement d̀ l'ensemble constituer par la Correspondance avec Élisabeth et le Traité des Passions de l'Aime, alors qu'il existe d'autres textes cartésiens faisant allusion ou référence au théâtre.? Bien plus, dans l'ensemble que nous venons de définir, nous allons isoler un élément, commencer par l'ítude de celui-ci et quasiment nous y arrêter: il s'agit de la première lettre a Élisabeth, ou Descartes décrit et utilise l'expérience du spectateur au théâtre. ${ }^{3}$ Enfin, nous n'aftirmons pas que la lecture, que nous proposons de ce texte premier, vaut pour tous les autres textes cartésiens sur le thesâtre: il se pourrait au contraire qu'il soit exceptionnel. En effet, cette lettre du 18 Mai 1645 permet de rapprocher et de confronter le théâtre et la métaphysiqute, alors que, par un autre versant, le théâtre conduit Descartes a la générosité, dans le Traité des Passions. Ce second versant, qui est sans doute principal pour Descartes lui-même, nous le contoumons, pour tenter d'explorer l'autre, même moins étendu et moins ensoleillé.

Rappelons rapidement le cadre de cette Lettre à Élisabeth du 18 Mai 1645 et de la première réfírence au théâtre qui y trouve place: Élisabeth a été longuement malade, voire l'est encore, d'une fièvre lente. Descartes, qu'elle a promu, dès le début de leur correspondance, 4 "meilleur médecin de son âme", mais qui a également proposé son avis à l'occasion d'un mal physique de la Princesse, ${ }^{5}$ donne a nouveau son opinion: "La cause la plus ordinaire de la fievre lente est la tristesse". Le mal du corps, donc, vient de l'âme et l'âme d'Élisabeth est elleméme malade des malheurs politiques de la famille Palatine. Il faut qu'Élisabeth "par la force de sa vertu, (rende son) âme contente, malgré les disgrâces de la fortune". Ici, pour préciser ce projet évidemment paradoxal et aider la Princesse a le réaliser, Descartes fait intervenir la distinction entre "les plus grandes Ames" et "celles qui sont basses et vulgaires". Ce qui caractérise les premières et les rend plus grandes que le commun des âmes, si l'on peut dire, nous l'appellerons l'asymétrie entre le bonheur et le malheur: les Ames basses, dit Descartes, "ne sont heureuses ou malheureuses, que selon que les choses qui leur surviennent sont agreables ou désagréables": elles coïincident immédiatement avec leurs passions, auxquelles "elles se laissent aller", passions qui ne sont elles-mèmes que l'effet brut des événements extérieurs, en tant qu'ils favorisent ou contrarient leurs désirs. A l'inverse, les grandes àmes retravaillent leurs passions: elles sont heureuses, lorsque les choses qui leur surviennent sont agréables, ${ }^{6}$ et,

\footnotetext{
1 "Les Fourberies du Malin Genie" et autres pieces.

2 J'en mentionnerai simplement un, qui témoigne d'un intêrit ancien: les premières lignes de l'Abregé de Ahsigne.

3 Lettre du 18 Mai 1645.

4 I6 Mai 16-43.

5 Juiilet $16-14$.

6 Mais, dit Descartes, tout en restant toujours maitresses de cette joie, de liçon à n"en être point "enivrêes": toutes les passions, y compris les gaies, sont bien retravaillées.
} 
surtout, car plus extraordinaire, "leur raison fait que leurs afllictions mème leur servent ct contribuent à la parfaite félicité dont elles jouissent dès cette vie". Voilà l'asymétrie, chez les grandes âmes, du bonheur et du malheur: elles se rendent toujours heureuses, directement par les succès de la fortune - tout en évitant, à nouveau, l'ivresse-, indirectement par ses revers. C'est dans ce cadre qu'intervient, quelques lignes plus loin, l'expérience théâtrale, comme réalisation exemplaire de ce "contentement de l'âme malgré les disgrâces de la fortune". Mais quelques lignes plus loin seulement: car Descartes donne d'abord une explication métaphysique de la force des grandes âmes: " $D$ 'une part, se considerant comme immortelles et capables de recevoir de très grand contentements, puis, d'autre part, considérant qu'elles sont jointes à des corps mortels et fragiles, qui sont sujets a beaucoup d'infirmités, et qui ne peuvent manquer de périr dans peu d'années, elles font bien tout ce qui est en leur pouvoir pour se rendre la fortune favorable en celte vie, mais néanmoins, elles l'estiment si peu, au regard de l'ćternité, qu'elles n'en considèrent quasi les événements que comme nous faisons ceux des comédies". La fonction de la métaphysique semble done double: d'une part, procurer à l'âme grande une jouissance particulière, image dès cette vie de la félicité future, après la mort, à contempler Dieu. Nous pouvons penser ici aux toutes dernières lignes de la Troisième Méditation: "comme la foi nous apprend que la souveraine félicité de l'autre vie ne consiste que dans cette contemplation de la Majesté divine, ainsi expérimenterons-nous dès maintenant, qu'une semblable méditation, quoique incomparablement moins parfaite, nous fait jouir du plus grand contentement que nous soyons capables de ressentir en cette vie". Dis cette vie, donc, cette jouissance métaphysique est la plus haute de toutes et les grandes âmes la connaissent. D'autre part, dit alors la lettre, protégées par ce contentement, elles ne soulfirent pas des malheurs de la fortune. Or, il me semble que l'on peut faire à cette détermination métaphysique de la grandeur d'hme deux objections, en ce qu'elle ne remplit pas parfaitement la promesse initiale de Descartes: en premier lieu, la jouissance métaphysique permet à l'âme d'éprouver du plaisir malgré les revers extérieurs, mais on ne peut dire alors que ceux-ci "servent" à cette félicité ou y "contribuent". En second lieu, et conséquence de la première objection, la grande âne ne risque-t-elle pas de devenir insensible, non seulement aux événements désagréâbables, puisqu'elle n'en souftre pas, mais aussi aux événements agréables, qu'elle finirait par estimer aussi peu que les malheurs, leur préférant le plaisir de l'âme seule à contempler Dieu? L'importance de l'expérience theátrale apparait alors: ce serait elle qui permettrait de comprendre vraiment comment les grandes âmes se rendent toujours heureuses, en corrigeant l'interprétation métaphy'sique. "Et comme les histoires tristes et lamentables, que nous voyons représenter sur un théâtre, nous donnent souvent aussi de récréation que les gaies, bien qu'elles tirent des larmes de nos yeux; ainsi ces plus grandes âmes, dont je parle, ont de la satisfaction, en elles-mémes, de toutes les choses qui leur arrivent, même les plus fầcheuses et insupportables." Deux types de pibces, donc: les comédies, ou "histoires gaies", dont les personnages sont heureux, au dénouement du moins, les tragédies ou "histoires tristes et lamentables", ou les personnages souffrent: les premières, nous le savons, s'achèvent souvent par des mariages, les secondes par la mort. Le spectateur de comédies, dit Descartes, est heureux: il se "récrée", sans doute parce qu'il prend plaisir et participe au bonheur des personnages. Le spectateur de tragidies, symétriquement, participe à la souffrance des héros: il compatit et pleure. Mais, dans le mème temps qu'il compatit et pleure, il prend plaisir. 11 s'agit ici d'un pur constat de Descartes: telle est l'expérience que nous faisons tous, lorsque nous assistons â une tragédie au théâtre. C'est dans des lettres ultérietures qu'Élisabeth et Descartes s'interrogeront sur l'origine de ce plaisir tragique. L'important est donc ailleurs: le plaisir du spectateur de tragédie nait de la souffrance, que lui inspirent les 
mallheurs des personnages, et ce modble apparait alors comme le meilleur pour la thèse initiale de Descartes: les grandes âmes sont heureuses des malheurs de la fortune comme de ses succès. En effet, le plaisir tragique répond â la première objection que l'on pouvait faire à l'argument métaphysique; par la pensée que le corps et l'âme sont distincts et que celle-ci est immortelle et appelée à contempler Dieu, contemplation dont elle peut jouir, sous une forme imparfaite dès cette vie, on peut bien éprouver du plaisir intèrieurement malgré la souftrance extérieure, due, elle, â l'union de l'âme et du corps. Mais le spectateur de tragédie éprouve du plaisir grâce à sa compassion pour les personnages; or, c'est bien cela que Descartes affirmait des grandes âmes: meme lorsque les événements sont défavorables et qu'elles en soullirent, elles parviennent à être heureuses de cela mẻme. Le plaisir tragique répond également à la seconde objection que l'on pouvait faire â l'interprétation métaphysique de la grandeur d'âme: celui qui, pour ne pas souffrir des malheurs de la vie, se retire d'elle pour méditer sur Dieu ou contempler sa majestś, celui-là risque de devenir insensible à la vie tout entière, non seulement à ses souflrances, mais aussi à ses bonleurs, parce qu'il se serait endurci et rendu indifférent aux événements de cette vie, bons ou mauvais, leur préfërant, dis maintenant, les bonheurs de l'âme seule. Au contraire, le spectateur de thểtre est un être que nous dirions hypersensible:7 il prend plaisir aux événements heureux des comédies et, loin d'être indifférent aux malleurs représentés dans la tragedie, y participe par compassion. Et, de fait, nous savons tous que, si la tragédie est mauvaise ou que le tragédien ne parvient pas â nous intéresser aux malheurs de son personnage, nous, spectateurs, nous ne nous apitoierons pas et, par conséquent, nous ne tirerons non plus aucun contentement du spectacle. Lintérêt du theâtre pour Descartes serait ainsi qu'il représente une expérience de l'entre-deux: le spectateur doit s'identifier au personnage, sans quoi pas de compassion ni de plaisir, mais cette identification n'y est jamais totale; il se trouve qu'il éprouve un certain contentement, en méme temps qu'il souftire pour le pauvre Rodrigue. S'il n'y avait que compassion et soullrance, le spectateur, selon Descartes, $n$ "irait pas au théâtre. De la même façon, les gens à qui les films d'horreur font seulement peur, par exemple, ne les regardent pas: vont voir ces films ceux, qui prennent plaisir à leur peur. Etre spectateur, selon Descartes, est donc toujours simultantment identification et distanciation; or, cette expérience conjointe du faire-un avec le personnage, sans quoi, encore une fois, le spectateur s'ennuie, et de l'être-distinct - aucun spectateur ne désire seulement compatir- nous semble plaire à Descartes, parce qu'il y voit un modkle exact de ce que devrait être la bonne relation entre l'âme immortelle et le corps auquel elle est jointe pendant cette vie, autrement dit, la msthode précise à suivre pour se rendre heureux en celte vie.

Nous faisons référence ici à la Lettre da 28 Juin 1643. la deuxième grande lettre de Descartes ì Ėlisabetli: "Ceux qui ne philosophent jamais, et qui ne se servent que de leurs sens, ne doutent point que l'âme ne meuve le corps, et que le corps n'agisse sur l'âme; mais ils considerent l'un et l"autre comme une seule chose, c'est-â-dire, ils conçoivent leur union; car concevoir l'union qui est entre deux choses, cest les concevair comme une seule. Et les pensées métaphysiques, qui exercent l'entendement pur, servent à nous rendre la notion de l’âme familiere; et l'étude des mathématiques, qui exerce principalement l'imagination en la considération des figures et des mouvements, nous accoutume à former des notions du corps bien distincles; et enlin, c'est en usant seulement de la vie ét des conversations ordinaires, el en s'abstenant de méditer et d"éludier allx choses gui exercent l'imagination, qu'on apprend â concevoir l'union de

7 A rapprocher de l'idéc cantisienme, selon laquelle les grandes $A$ mes ont, en vérité, des passions plas fortes qus les autres. 
l'Ame et du corps". Comprendre l'union de l'âme et du corps ne fait problème que pour ceux qui ont fait, au moins une fois en leur vie, de la métaphysique et compris leur réelle distinction: c'est le cas d'Élisabeth au début de sa correspondance avec Descartes. Mais cette difliculté est richesse, car, désormais, on peut essayer de vivre en connaissance de cause, si j'ose dire; l'union de l'âme et du corps pourra être réellement, c'est-A-dire toujours, heureuse, pour les àmes qui se savent en même temps distinctes, et pour celles-là seules; et ce sont elles que Descartes dira grandes. D'ou cette réflexion, le 28 Juin 1643, sur l'emploi du temps: s'il suffit d'employer fort peu d'lıeures, par an, à la métaphysique, entendons â la métaphysique pour elle-même, c'est parce qu'il s'agit maintenant de se rappeler les conclusions de cette métaphysique, $d$ '“employer" les vérités qu'elle a permis de connaitre tout le reste du temps, tout le temps de la vie. Pour vivre heureux, il faut vivre, et non faire de la métaphysique; mais il faut vivre du point de vue de la métaphysique. Telle serait done l'originalité et l'inportance de l'argument thêâtral, en sa première occurrence entre Descartes et Elisabeth: corriger le discours d'abord métaphysique, par lequel Descartes propose â la Princesse un moyen d'être heureuse, quelles que soient les diflicultés extérieures. Nous proposerons une troisième diffërence entre métaphysique et thêâtre: le modèle théâtral de la grandeur d'äme semble plus universel que la détermination métaplyssique de celteci, parce qu'il est laic. L'expérience du plaisir tragique est partagée par tous, et l'on pourrait inaginer alors, à la limite, une grande âme qui soit athée, ce qui est en revanche inconcevable dans la première caractérisation cartèsienne du bonheur intérieur. Le sort des athées, dira-t-on, indiffère sans doute Descartes ${ }^{8}$ et ce n'est pas pour eux qu'il fait référence au thèatre; mais ce n'est pas si simple peut-être, car, si Descartes et Élisabeth partagent la mẻme foi en une vie future de félicité parfaite dans la contemplation de Dieu, le philosophe est catholique et la Princesse protestante, différence de confession qui sera cause, au moins une fois dans leur correspondance, d'une brouille assez sérieuse, une des seules et peut-être la plus nette dans les lettres que nous connais. sons. En effet, le 30 Novembre 1645, Élisabeth scrit à Descartes à propos de l'abjuration et de la conversion à l'Église Romaine de son frère Édouard: "Cette folie", dit-elle, " $m$ 'a plus troublé la santé du corps et la tranquillité de l'Ame que tous les malheurs qui me sont encore arrivés". La grandeur d'âme d'Élisabetll, semble-t+il, n'a pasété ici suffisante. Mais surtout, elle ajoute quelques phrases, évidemment difficiles a lire pour Descartes: "Il faut qque je voie une personne, que j'aimais avec autant de tendresse que j'en saurais avoir, abandonnée au mépris du monde et $\grave{a}$ la perte de son âme (selon ma croyance). Si vous n'aviez plus de charité que de bigoterie, ce serait une impertinence de vous entretenir de cette matière (....)". Malgré ces précautions tardives, la réponse de Descartes se fera attendre et ne manquera pas de froideur: "Je ne puis nier que je n'aie été surpris d'apprendre que Votre Altesse ait eu de la fâcherie, jusqu'au en être incommodée pour sa santé, pour une chose que la plus grande part du monde trouvera bonne (...)".9 Or, dans cette même lettre, ayant rapidement laissé de cété ce sujet épineux, Descartes répond à Élisabeth sur une question qui est une reprise exacte, sous une forme dilférente, de celle des grandes âmes: avons-nous bien dans la vie, comme vous le dites, a demandé la Princesse, ${ }^{10}$ plus de biens que de maux? Or, on verrait mal Descartes, juste après l'bvidence, simplement refoulée, de leur divergence de foi, reprendre alors l'argumentation métaphysique de la Lettre du 18 Mai 1645; voici le début de sa réponse: "Ce qui m'a fait dire qu'il y a toujours plus de biens que de

8 On sait, également, que les atháes ne sauraient Otre mathématiciens, selon lui; c'est pourquai nous imaginons, presque comme une boutsde, une grande atme athere.

9 Janvier $1 \mathrm{~B}+6$.

1030 Novembre 1645. 
maux en cette vie, c'est le peu d'état que je crois que nous devons faire de toutes les choses qui sont hors de nous, et qui ne dépendent point de notre libre- arbitre, à comparaison de celles qui en dépendent, lesquelles nous pouvons toujours rendre bonnes, lorsque nous en savons bien user". Pourtant, la métaphysique n'est pas si loin: "laire peu d'état des choses hors de nous" nous rappelle le peu d'estime pour les choses de fortune, dans la Lette du $18 \mathrm{Mai} 1645$; et le libre-arbitre, dont le bon usage permet de jouir toujours du bien, ne renvoie-t-il pas directement à la distinction de l'Ame et du corps'? Il faudrait montrer ici que, si la vertu cartésienne s'identifie d'abord à la résolution de suivre le bien (représenté par l'entendement), cetté résolution constituant précisément le bon usage du libre-arbitre, celui-ci ne saurnit rester purement formel et doit inclure le plus possible la connaissance du vrai bien: nous pensons en particulier ici à la Lettre de Descartes, le 15 Septembre 1645, ou, toujours sur la demande d'Élisabeth," Descartes énonce les vérités qu'il nous est utile de connaitre: or, les deux premières sont à nouveau l'existence, puissance et perfection de Dieu, d'une part, la distinction de l'Ame et du corps, d'autre part. Simplement, il nous semble qu'en Janvier 1646, bien qu'Élisabeth vienne de vivre une des épreuves les plus difficiles de sa vie et qu'il soit d'autant plus urgent que Descartes l'aide à se rendre heureuse-enquelque-fagon-malgré-tout, il ne saurait arguer de la foi en Dieu et en l'immortalité de l'âme. Car, comme l'a revélé l'incident provoqué par la conversion d'Édouard, leurs fois respectives restent sans doute irréductiblement différentes. Ainsi, Descartes se réfbre en ces circonstances au thếtre seul; voici la suite de la Lettre de Janvier 1646: "et nous pouvons empêcher, par leur moyen (c'est-ì-dire, en rendant toujours bonnes les choses qui dépendent de notre libre-arbitre), que tous les maux qui viennent d'ailleurs, tant grands qu'ils puissent être, n'entrent plus avant en notre Ame gue la tristesse que y excitent les combdiens, lorsqu'ils représentent devant nous quelques actions fort funestes; mais j'avoue, ajoute Descartes, qu'il faut être fort philosophe pour arriver jusqu'au ce point". La philosophie par le théâtre est peut-être difficile, mais elle est au moins universelle, parce que la'ique. Nous reconnaissons, en vérité, que la Lettre du 18 Mai 1645 , la premibre, done, ou apparait le thẻme du théâtre, est aussi, $A$ notre connaissance du moins, la seule ou soient directement rapprochés le modèle méthaphysique et le modèle théâtral de la grandeur d'âme, rapprochement qui nous a autorisé à les confronter. Dès lors, dans les lettres que nous venons d'examiner, l'échange, de Novembre 1645 a Janvier 1646, autour de la conversion du frère d'Élisabeth, la rélbrence de Descartes à l'expérience théâtrale seule, à l'exclusion de toute exhortation métaphysique, peut sembler elle-mème peu significative; nous croyons cependant que le contexte religieux dramatique donne ici un certain piment a cette séparation des deux registres. En revanche, l'autonomie génèrale de la reférence au théatre nous semble signaler que sa fonction ne se réduit pas à celle que nous avons isolée ici, à savoir, corriger la détermination purement métaphysique du bonlıur véritable. En particulier, il s'y joute certainement aussi. et sans doute plus essentietlement pour Descartes lui-mėme, la pensée d'autrui et de mon attitude face aux souffrances d'autrui. Il est très significatif que, dans le Traité des Passions, les deux principales occurrences du thbme du plaisir tragique'2 aient pour objet la fagon, dont la grande âme, ou maintenant l'âme gènéreuse, doit se comporter au regard du malheur des autres homenes: comment peut-elle y participer, compatir, tout en éprouvant à cette occasion une satisfaction inté-

11 Août 1645; Elisabeth à Descantes; "j'espière qut vous continuerez a m'enseigner les inoyens de fortilicr l'entendement, pour juger du meilleur en toutes les actions de la vie, qui me semble ŝtre la seule di!jiculté, puisqu'il est impossible de ne point suive le bon chemin. quand il est connu".

12 Dans les articles 147, "Dess émations intéricures de l'âme”, et 187, "Comment les plus génhreux sont touchés de la pitič". 
rieure? Denis Kambouchner, dans L'Homme des Passions, ${ }^{13}$ a donné de ces pages un beau commentaire, montrant par exemple que la figure du "veuf joyeux", qui précéde, A l'article 147, la référence au théâtre clle-même, n'était en rien immorale. Restent cependant, croyons-nous, deux questions, une pour chaque lecture possible du thême du théâtre. Si, comme nous avons essayé de l'envisager, le modelle théâtral est une autre faqon pour se rendre heureux, soi, dans les malheurs qui surviennent, modble autre que la mètaphysique ou la foi, le problème ne se pose-t-il pas, évident et immédiat peut-être, essentiel néanmoins, de sa mise en pratique'? Pour le dire brutalement, lorsque j'aurai vu sur une scêne Rodrigue confronté à un choix tragique, "triste et lamentable" comme dit Descartes, entre l'honneur de sa famille et son amour pour Chimhne, que j'aurai compati à sa souftrance et, par ll-méme, pris plaisir au spectacle, serai-je, moi, capable de moins souffrir, au cas ou je me trouverais dans une situation analogue, fait-elle moins triste et lamentable? Plus généralement, voir reprbsenter des passions tristes sur un thèhtre provoque en nous compassion et plaisir, selon Descartes, et non plus terreur et pitit; mais faut-il penser alors qừ l'expérience théâtrale, en tant que telle, nous prépare à résister à la réalité des passions, à n'en pas souffrir et à vivre heureux malgré elles? Il faudrait penser et développer alors une pédagogie par le théâtre, par la littérature, ou même par l'ar en gẻnéral. Revenons enlin à l'autre signification de l'expérience théâtrale chez. Descartes, sans doute sa signilication principale - meis nous avons tenté d'en exposer une, marginale, entre les lignes de la Lettre du 18 Mai 1645-, illustrer, non plus directement la grandeur d'âme face aux revers de fontune, nais la générosité face aux malheurs d'autrui, malheurs d'autrui qui sont cependant susceptibles de m'atteindre et de me faite souffrir indirectement; la question n'est-elle pas encore celle de la mise en pratique de ce modsle, mais sous une forme plus particulière? Dans la vie réelle, confronté à la souffrance des autres, et d'autant plus que ces autres me sont plus proches, comment ne pas compatir jusqu'au bout, si l'on peut dire, sans pouvoir se détacher de cette souffrance paragce avec autrui et sans pouvoir, par conséquent, éprouver de contentement intérieur? C'est une des questions permanentes d'Élisabeth à Descartes: jusqu'ou doit-clle faire siens les malheurs politiques de sa famille? Comment ne pas y participer de tout son être et, ainsi, n'en tirer que souffrance? Comment trouver une limite à son engagement, alors que ces étres, pensons par exemple à ses frères, dont l'un se convertit, l'autre tue un gentilhomme en duel, fui sont absolument chers, alors qu'elle les aime avec autant de tendresse qu'clle en saurait avoir, selon les termes de sa Lettre du 30 Novembre 1646? Ou encore, comme le montre bien Denis Kambouchner, il est essentiel que le "veuf joyeux" de l'article 147 du Traité des Passions n'ait pas aimé sa femme, de telle façon qu'il puisse, tout en ctant touché de sa mort, éprouver cette "joie secréte dans le plus intérieur de son âme", qui n'a, encore une fois, rien d'immoral, quasiment comme, à voir mourir sur scène un personnage de théâtrè, on est touché de pitie, mais aussi secrétement heureux: simplement, qu'en serait-il du veul's'il avait follement aimé sa femme? Pensons également â ce que Descartes dit de la mort de sa propre fille, Francine. Dilliçulté de vivre comme au théâtre.

13 Paris, Albin Michef, 1996. 